\title{
DATA ON THE EPIDEMIOLOGY AND PATHOLOGY OF ANATIPESTIFER DISEASE IN HUNGARY (2010-2014)
}

\author{
Éva GYURIS ${ }^{1}$, Csaba NEMES ${ }^{2}$ and Tibor MAGYAR ${ }^{3 *}$ \\ ${ }^{1}$ Laboratory of Domestic Mammal, Wildlife and Poultry Diseases, National Food Chain \\ Safety Office - Veterinary Diagnostic Directorate, Budapest, Hungary; ${ }^{2}$ Veterinary \\ Diagnostic Laboratory in Kaposvár, National Food Chain Safety Office - Veterinary \\ Diagnostic Directorate, Kaposvár, Hungary; ${ }^{3}$ Institute for Veterinary Medical Research, \\ Centre for Agricultural Research, Hungarian Academy of Sciences, P.O. Box 18, \\ H-1581 Budapest, Hungary
}

(Received 9 April 2018; accepted 25 July 2018)

\begin{abstract}
Anatipestifer disease is a contagious disease caused by Riemerella anatipestifer, affecting primarily ducks, geese and turkeys, and characterised by listlessness, diarrhoea, sneezing, nasal discharge, and nervous signs. Sporadically, it occurs in a wide range of other domesticated and wild birds as well. The incidence and characteristics of the disease seen in the three main host species are summarised based on birds submitted for routine laboratory investigation in Hungary over the period 2010-2014. The infection was diagnosed in a higher percentage in geese $(9.9 \%)$ and ducks $(7.5 \%)$. It occurred in 5-day-old to 17 -week-old geese and 3- to 6.5-week-old ducks, respectively. The pathological lesions were comparable in these two species: enlarged spleen, serofibrinous pericarditis, perihepatitis, airsacculitis, catarrhal enteritis, subcutaneous oedema and hyperaemia over the cranium, mucopurulent exudate in the nasal cavity and occasionally pneumonia, conjunctivitis, purulent arthritis and caseous salpingitis. In some cases, $R$. anatipestifer produced only secondary lesions, which complicated other diseases such as circovirus infection, mycotoxicosis, mycoplasmosis, or Derzsy's disease. In turkeys, the disease occurred rarely $(0.5 \%)$ and at an older age (12 to 19 weeks). The lesions most frequently seen were purulent osteomyelitis of the cranium and seropurulent meningitis. Purulent osteomyelitis in the cranium caused by $R$. anatipestifer infection had not been reported in turkeys previously. To various extents, other local lesions such as serofibrinous pericarditis, airsacculitis, arthritis, and in one case septicaemia were also observed. The high incidence of the disease in waterfowl underlines the importance of appropriate treatment and prevention that should be based on accurate diagnosis and antimicrobial susceptibility testing, proper biosecurity and vaccination with regard to the serotype(s) present on the farm.
\end{abstract} turkey

Key words: Anatipestifer disease, Riemerella anatipestifer, goose, duck,

\footnotetext{
*Corresponding author; E-mail: magyar.tibor@agrar.mta.hu; Phone: 0036 (1) 467-4092
} 
Anatipestifer disease was first described in geese as 'septicaemia anserum exsudativa' in Germany by Riemer (1904). In ducks, the disease was first diagnosed by Hendrickson and Hilbert (1932). They also isolated and characterised the causative bacterium, and called it Pfeifferella anatipestifer. It is a Gramnegative, rod-shaped, non-motile, non-spore-forming bacterium that had been reclassified several times (Moraxella, Pasteurella) until finally it was moved to the family Flavobacteriaceae and, in honour of Riemer, ultimately renamed as Riemerella anatipestifer (Segers et al., 1993). In Hungary, anatipestifer disease was first reported in ducks (Bitay et al., 1979), and almost twenty years later in geese as well (Ivanics et al., 1996).

Riemerella anatipestifer infection occurs all over the world where intensive duck or goose production is practised. It causes severe economic losses through high mortality rate (estimated to vary from $10 \%$ to $75 \%$ ), weight loss, the costs of antibiotic treatment, prevention, and condemnation at the slaughterhouse (Ruiz and Sandhu, 2013).

Anatipestifer disease is observed most frequently in young (2- to 8-weekold) ducklings and goslings, characterised by listlessness, diarrhoea, upper respiratory signs and nervous signs such as head tremor, torticollis and lameness (Pickrell, 1966; Jortner et al., 1969; Pierce and Vorhies, 1973; Bisgaard et al., 2008; Fulton and Rimler, 2010). Birds are infected via the respiratory route or through skin wounds (Ruiz and Sandhu, 2013). Natural outbreaks have been described in 6- to 15-week-old turkeys as well (Zehr and Ostendorf, 1970; Smith et al., 1987; Frommer et al., 1990). Furthermore, sporadic cases of $R$. anatipestifer infections have also been reported in chickens, guinea fowl, pheasants, gulls, quails, budgerigars, and wild waterfowl (Bruner et al., 1970; Karstad et al., 1970; Rosenfeld, 1973; Pascucci et al., 1989; Hinz et al., 1998). Adverse environmental conditions (hot or cold weather, inadequate ventilation, stress caused by overcrowding) or concomitant diseases can predispose birds to disease outbreaks (Ruiz and Sandhu, 2013).

To date, no data have been published on the occurrence and characteristics of anatipestifer disease in different host species in Hungary. The aim of this study was to summarise the incidence and the lesions of anatipestifer disease observed in geese, ducks and turkeys in Hungary in the period of 2010-2014 based on the findings obtained in birds submitted for routine laboratory investigation.

\section{Materials and methods}

\section{Cases}

Birds from goose, duck and turkey flocks submitted for routine laboratory investigation to the Veterinary Diagnostic Directorate (Budapest, Kaposvár, Debrecen, Hungary) in the period 2010-2014 were included in the study. In total, the 
case number was 1418 for goslings, 411 for ducklings, and 1292 for turkeys (Fig. 1). Each case represented 4 to 10 birds that were submitted in one group from the same flock. The age of the birds ranged from 5 days up to 36 weeks (geese), up to 22 weeks (ducks), and up to 28 weeks (turkeys). Cases diagnosed with anatipestifer disease were the subject of this study. The goose cases originated from 84 flocks, and from 19 flocks more than one bird was submitted. The duck cases originated from 19 flocks, and from three flocks birds were received on several occasions. The turkey cases originated from four flocks, and samples from two flocks were obtained several times. The presence of other diseases was examined according to routine procedures; however, the details are not shown here. Only the diagnoses were used for calculating the incidence of concomitant infections in cases positive for anatipestifer disease.

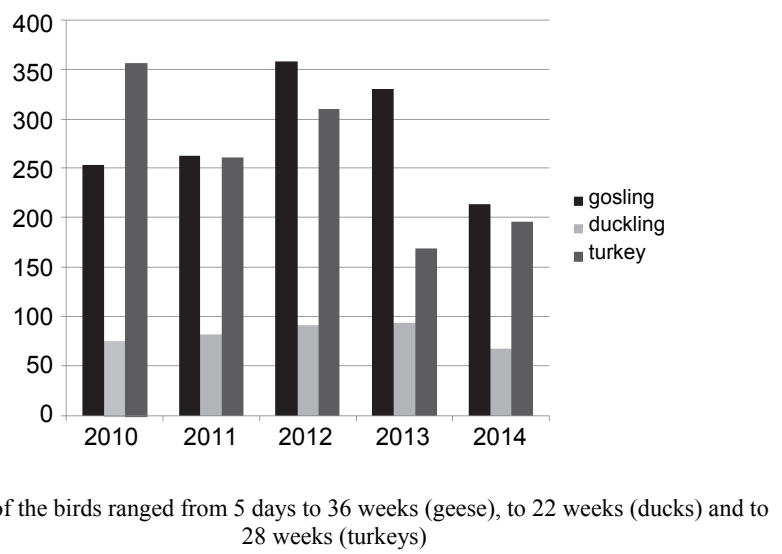

Fig. 1. Number of gosling, duckling and turkey cases ${ }^{*}$ submitted for routine laboratory investigation to the National Food Chain Safety Office - Veterinary Diagnostic Directorate (NÉBIH-ÁDI, Budapest, Debrecen, Kaposvár) in the years 2010-2014

\section{Postmortem examination}

After detailed gross pathological examination of the birds, representative tissue samples were collected for histopathological examination in each case. Specimens were generally taken from the brain and liver, occasionally from the heart, bursa of Fabricius, lung, spleen, kidney, spine, and cranium. Tissue samples were fixed in $10 \%$ neutral-buffered formalin, embedded in paraffin, sectioned at $4 \mu \mathrm{m}$ and stained with haematoxylin and eosin.

\section{Isolation of $R$. anatipestifer}

Various tissues, including the liver, pericardium, brain, lung, oviduct, joint and occasionally the trachea, conjunctiva, cranium, air sac and peritoneum were sampled, and all samples were cultured on Columbia agar plates (Biolab, Buda- 
pest, Hungary) supplemented with $5 \%$ sheep blood at $37{ }^{\circ} \mathrm{C}$ for $24 \mathrm{~h}$ in an atmosphere containing $5 \% \mathrm{CO}_{2}$. Riemerella anatipestifer suspect strains were first identified by colony morphology, oxidase reaction and Gram staining, and their identity was confirmed with a species-specific polymerase chain reaction (PCR) (Kardos et al., 2007).

\section{Results}

\section{Incidence and epidemiology}

Anatipestifer disease was diagnosed in 141 cases $(9.9 \%)$ in geese, and in 31 cases $(7.5 \%)$ in ducks. The disease was rather rare among turkeys. It was diagnosed only in seven cases (0.5\%) (Fig. 2).

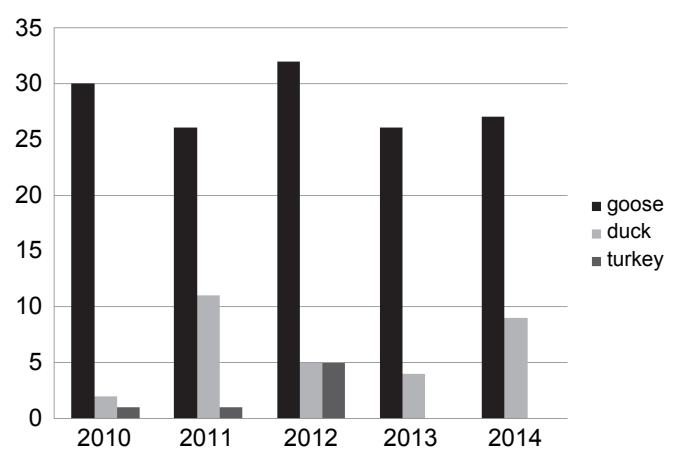

*Each case contained 4-10 birds submitted together for routine laboratory investigation

Fig. 2. Number of cases ${ }^{*}$ of anatipestifer disease diagnosed in geese, ducks and turkeys at the National Food Chain Safety Office - Veterinary Diagnostic Directorate (NÉBIH-ÁDI, Budapest, Debrecen, Kaposvár) in the years 2010-2014

In geese, the incidence of anatipestifer disease was comparable to that of Derzsy's disease, circovirus infection, haemorrhagic nephritis-enteritis (polyomavirus infection), colibacillosis, streptococcosis, and mycotoxicosis (indicated by the presence of tubulonephrosis and liver dystrophy). The case number of fowl cholera and paratyphoid infection was about half of the anatipestifer disease cases (Table 1).

In ducks, the incidence of anatipestifer disease, showing some variation in different years, was similar to that of fowl cholera, circovirus infection, colibacillosis, and mycotoxicosis (Table 2).

Riemerella anatipestifer infections were diagnosed most frequently in young, 1- to 8-week-old or more often in 2- to 5-week-old goslings, 3- to 6week-old ducklings and 12- to 19-week-old turkeys. The chronic form of anatipestifer disease accompanied by severe weight loss was rarely observed. 
Table 1

Incidence of diseases most commonly diagnosed in geese at the National Food Chain Safety Office - Veterinary Diagnostic Directorate (NÉBIH-ÁDI, Budapest, Debrecen, Kaposvár) in the years 2010-2014

\begin{tabular}{|c|c|c|c|c|c|}
\hline \multirow{2}{*}{ Diseases } & \multicolumn{5}{|c|}{ Years } \\
\hline & 2010 & 2011 & 2012 & 2013 & 2014 \\
\hline Derzsy's disease & $39^{*}$ & 44 & 64 & 53 & 29 \\
\hline Streptococcosis & 20 & 25 & 43 & 40 & 37 \\
\hline Colibacillosis & 19 & 16 & 22 & 33 & 46 \\
\hline Pneumomycosis & 12 & 34 & 25 & 33 & 14 \\
\hline Circovirus infection & 16 & 24 & 34 & 41 & 19 \\
\hline Anatipestifer disease & 30 & 26 & 32 & 26 & 27 \\
\hline Polyomavirus infection & 10 & 13 & 38 & 42 & 7 \\
\hline
\end{tabular}

${ }^{*}$ The number of positive cases

Table 2

Incidence of diseases most commonly diagnosed in ducks at the National Food Chain Safety Office - Veterinary Diagnostic Directorate (NÉBIH-ÁDI, Budapest, Debrecen, Kaposvár) in the years 2010-2014

\begin{tabular}{lccccc}
\hline & \multicolumn{5}{c}{ Years } \\
\cline { 2 - 5 } Diseases & 2010 & 2011 & 2012 & 2013 & 2014 \\
\hline Circovirus infection & $8^{*}$ & 13 & 14 & 28 & 9 \\
Mycotoxicosis & 10 & 9 & 8 & 6 & 11 \\
Fowl cholera & 10 & 9 & 7 & 9 & 5 \\
Colibacillosis & 5 & 10 & 9 & 7 & 9 \\
Pneumomycosis & 2 & 3 & 1 & 2 & 7 \\
Anatipestifer disease & $\mathbf{2}$ & $\mathbf{1 1}$ & $\mathbf{5}$ & $\mathbf{4}$ & $\mathbf{9}$ \\
\hline
\end{tabular}

${ }^{*}$ The number of positive cases

In geese, anatipestifer disease occurred at the age of 5 days to 17 weeks. In some cases, concurrent diseases were also observed in addition to anatipestifer disease in some of the birds belonging to the case in question. In $19 \%$ of the cases circovirus infection, while in 5\% of the cases Derzsy's disease was the primary diagnosis. In $21 \%$ of the cases some of the birds died of colibacillosis and in $12 \%$ of the cases of pneumomycosis, while in $8 \%$ of the cases each paratyphoid and streptococcosis occurred as concomitant diseases; there was only one case in which the majority of the birds died of anatipestifer disease.

In ducks, anatipestifer disease occurred at the age of 3 to 6.5 weeks. In some cases, concurrent diseases were diagnosed as well. In $46 \%, 13 \%$ and $8 \%$ of the cases circovirus infection, mycotoxicosis and mycoplasmosis was the primary diagnosis, respectively. In $38 \%$ of the cases some birds died of colibacillosis, 
but the majority of them succumbed to anatipestifer disease. Paratyphoid, staphylococcosis, pasteurellosis, amyloidosis, and visceral gout also occurred in a few birds within some cases.

In turkeys, anatipestifer disease occurred at the age of 12-19 weeks. Riemerella anatipestifer could be isolated in pure culture from local lesions of the cranium, meninges and joints. In one case, besides the afore-mentioned localisations, $R$. anatipestifer pericarditis and peritonitis were also seen. In another case, $R$. anatipestifer septicaemia was also observed. In $72 \%$ of the cases, local $R$. anatipestifer infection was accompanied by concurrent $E$. coli septicaemia. In some of these cases, $R$. anatipestifer could be isolated together with $E$. coli from certain locations such as the pericardium, air sacs and airways. Interestingly, although most of these cases occurred at different time points and at various geographic locations, all of the affected flocks belonged to the same owner.

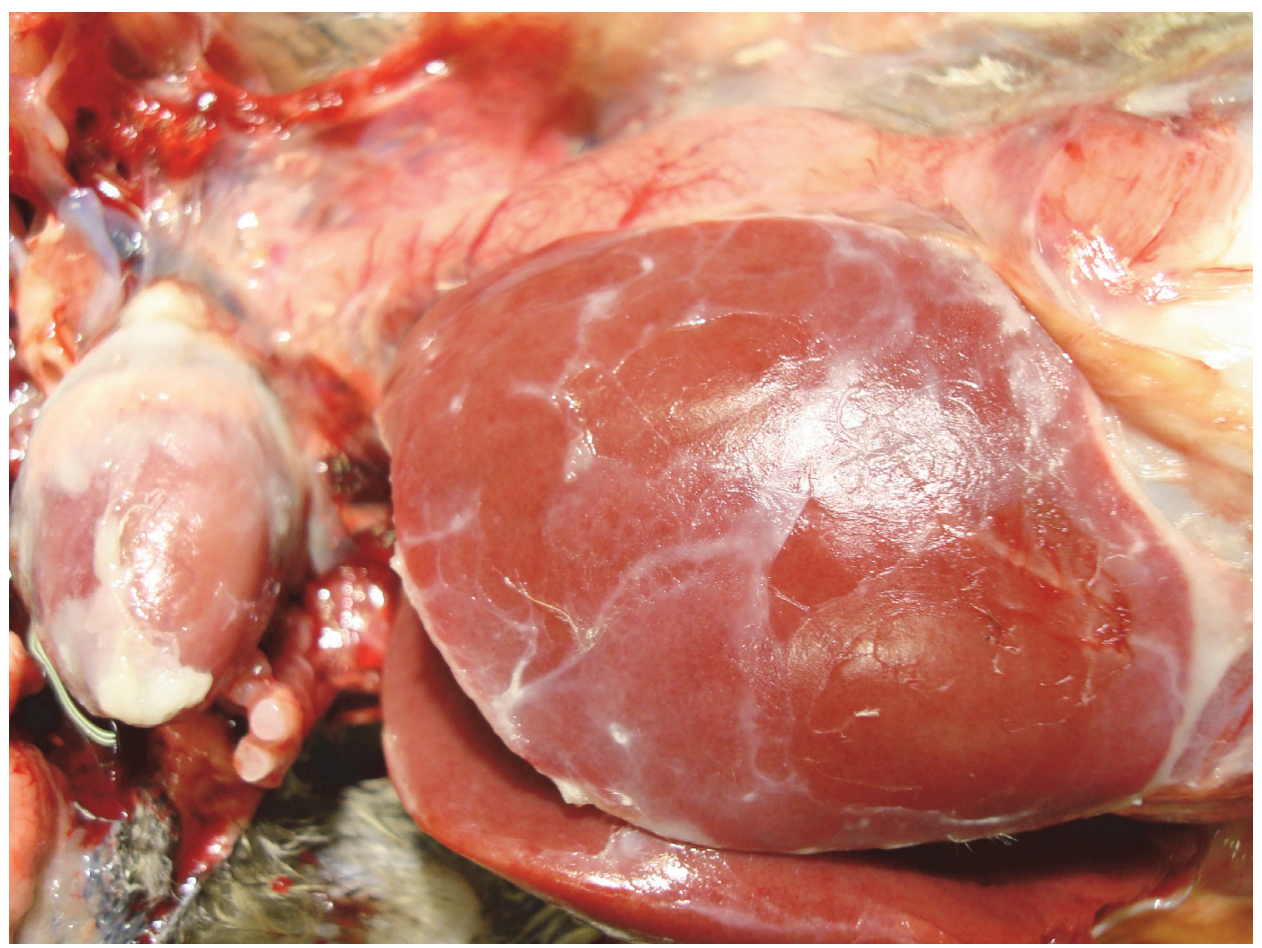

Fig. 3. Fibrinous exudate on the surface of the liver and on the pericardium in a goose

\section{Pathological lesions}

In geese and ducks, serofibrinous pericarditis, perihepatitis and airsacculitis were the most obvious gross lesions of $R$. anatipestifer infection. Fibrinous exudate accumulated in the pericardial sac, and on the surface of the liver (Fig. 3). 
The spleen was generally enlarged (Fig. 4), occasionally mottled, and congestion occurred in the liver. Mucopurulent exudate was seen in the nasal cavity and sinuses, and sporadic catarrhal pneumonia was also noticed. Acute catarrhal enteritis was seen in the small intestine. Usually some birds showed oedema and hyperaemia of the subcutaneous connective tissue over the cranium within each case (Fig. 5). Occasionally, inflammation of the joints or caseous salpingitis also occurred (Fig. 6). Serofibrinous arthritis occurred in $6 \%$ of the goose cases, caseous exudate was observed in the oviduct in 5\% of the goose cases and in $13 \%$ of the duck cases. Conjunctivitis was observed in $4 \%$ of the duck cases.

In most of the cases, $R$. anatipestifer infections caused only local lesions in turkeys. The most prominent lesion was cranial suppurative osteomyelitis and consequent seropurulent leptomeningitis. Rarely, seropurulent arthritis and pericarditis associated with $R$. anatipestifer infection were also observed. In a single case, $R$. anatipestifer could be isolated not only from local lesions but also from the heart blood (septicaemia). In several cases, although E. coli septicaemia/ bacteraemia could be determined from local lesions, $R$. anatipestifer was grown in pure culture.

\section{Histopathological lesions}

In geese, ducks and in two turkey cases, serofibrinous inflammation was observed on the epicardium (Fig. 7), on the Glisson's capsule of the liver, on the pleura, and in the wall of the air sacs. The exudate contained lymphocytes, histiocytes and heterophil granulocytes. Serous hepatitis was observed, the sinusoids of the liver were distended, and white blood cells accumulated between the liver plates. In some parenchymal cells of the liver hydropic degeneration was observed. Mild periportal lympho-histiocytic infiltration was seen as well. Occasionally in the lower respiratory tracts, in the parabronchi of the lung serous or catarrhal exudate was observed. Lymphoid depletion occurred in the medulla of the follicles in the bursa of Fabricius. Serofibrinous meningitis and ventriculitis were seen in the brain. The leptomeninges of the brain and spine were diffusely widened, serofibrinous inflammation and lymphocytic, histiocytic and heterophil granulocytic infiltration were seen (Fig. 8). Similar cellular infiltration occurred in the ventricles of brain, in the central canal of the spine and under the ependyma as well. In the majority of the turkey cases signs of purulent osteomyelitis with heterophil granulocytic infiltration were observed in the cranium (Fig. 9), and this lesion was regularly associated with seropurulent meningitis. The leptomeninx of the brain was widened, seropurulent inflammation was seen with mostly heterophil granulocytic and also lymphocytic and histiocytic infiltration. 


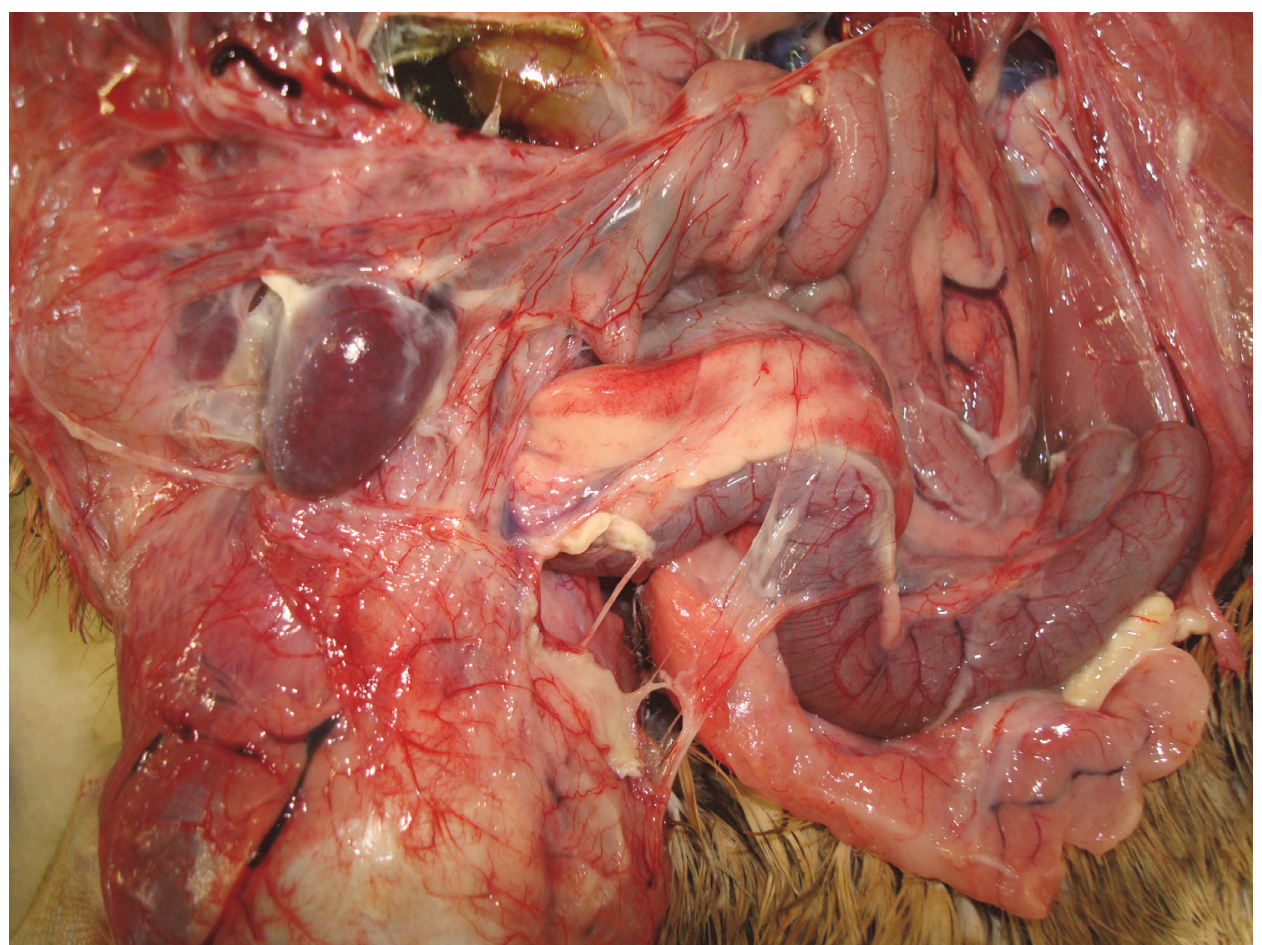

Fig. 4. Enlargement of the spleen and serofibrinous inflammation in the body cavity of a goose

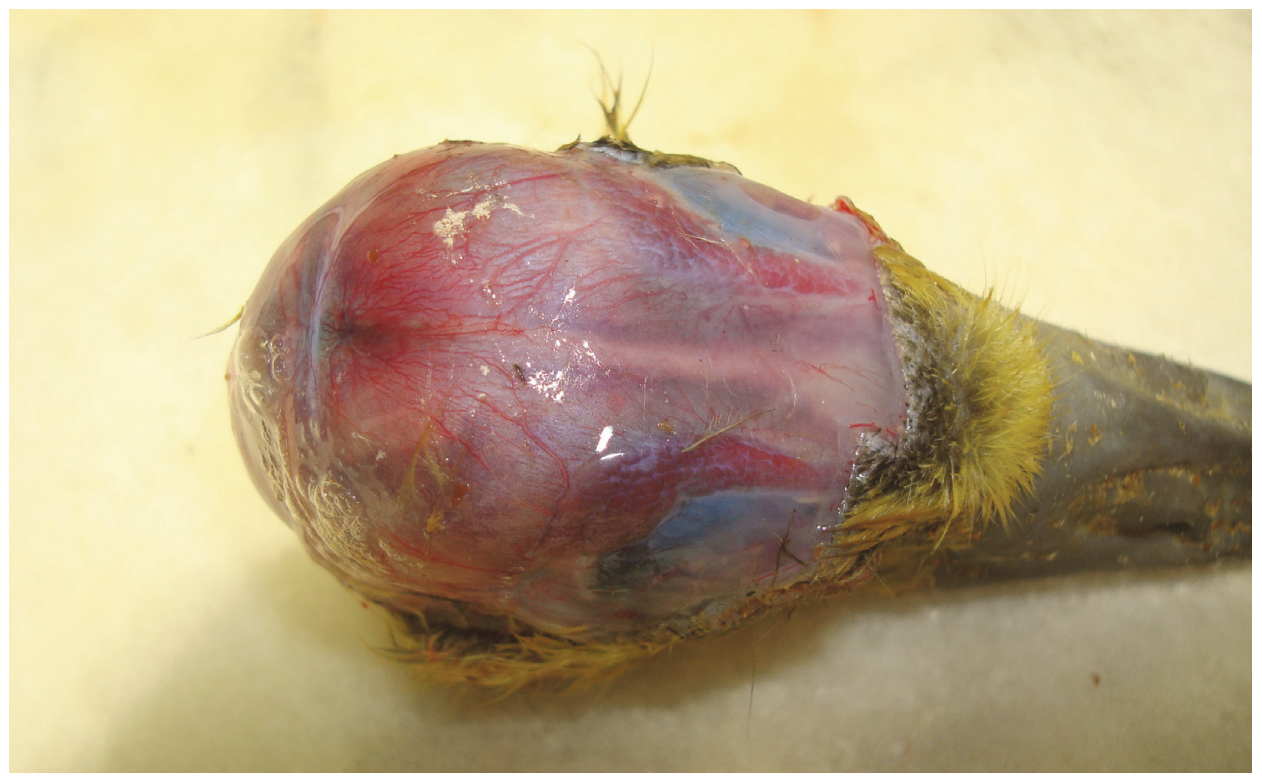

Fig. 5. Oedema and hyperaemia of the subcutaneous connective tissue over the cranium in a goose 


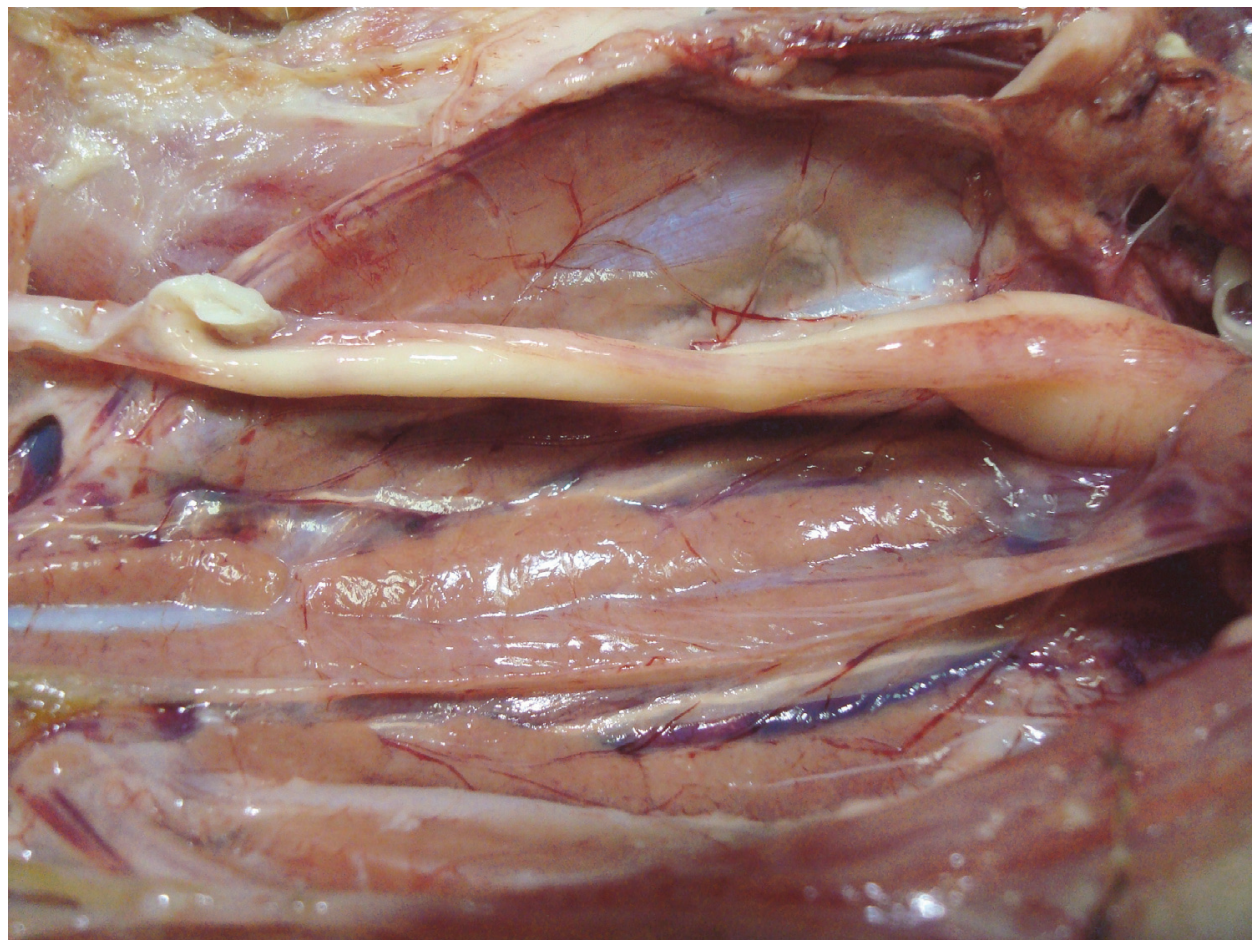

Fig. 6. Caseous salpingitis in a goose

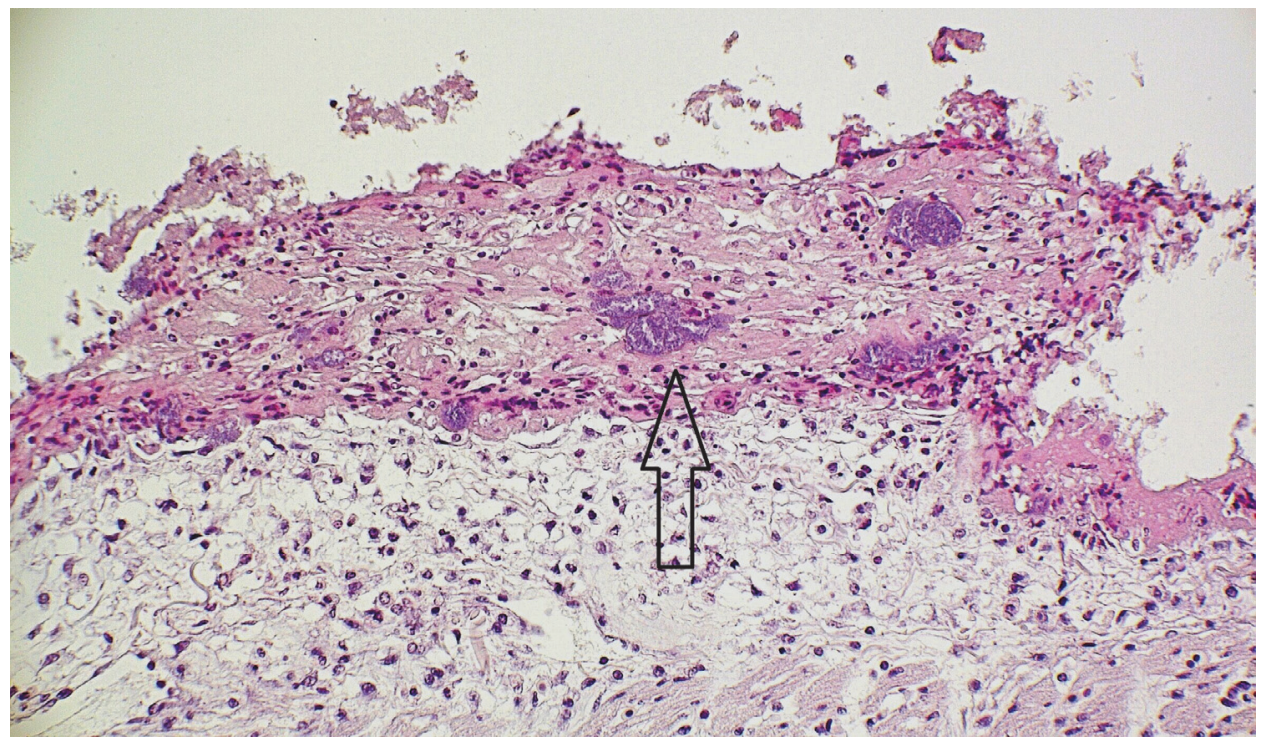

Fig. 7. Serofibrinous inflammation in the epicardium with clusters of bacteria $(\uparrow)$ in a goose. Haematoxylin and eosin, $\times 400$ 


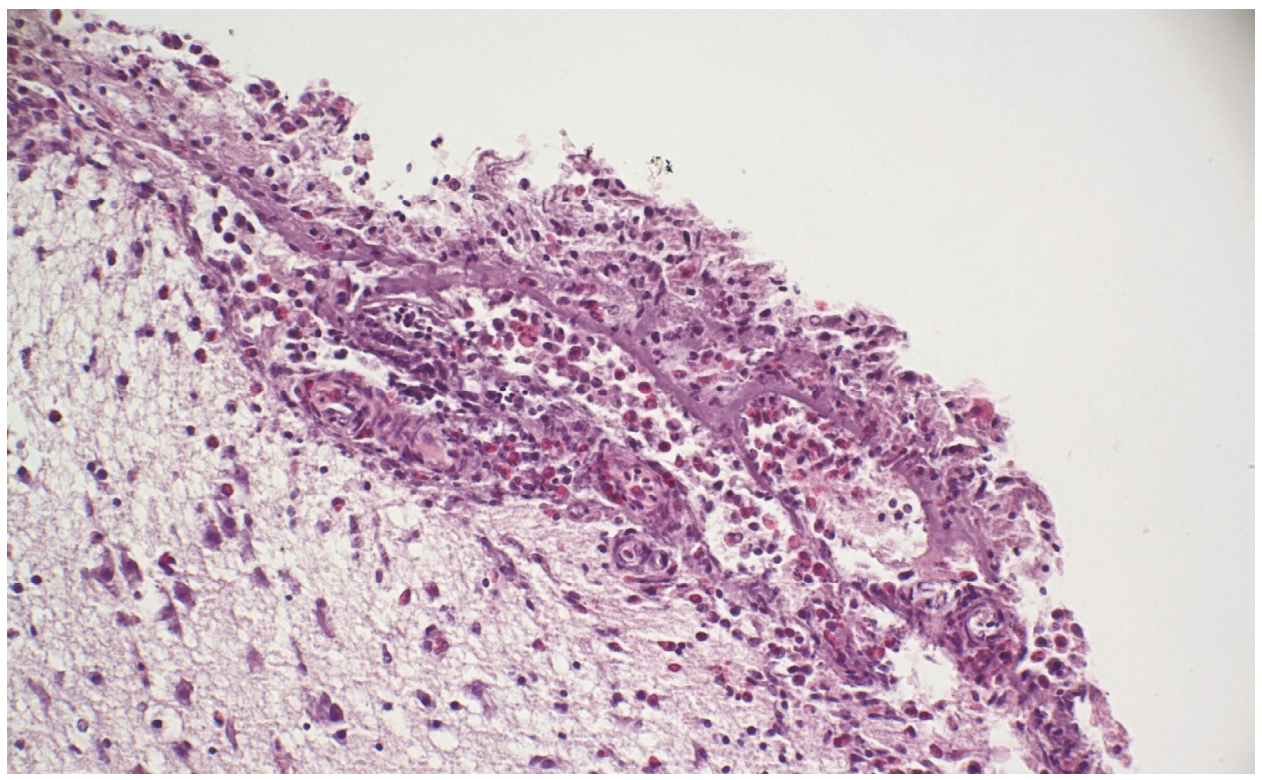

Fig. 8. The leptomeninx of the brain is diffusely thickened and infiltrated with inflammatory cells (lymphocytes, histiocytes and heterophil granulocytes) in a goose. Haematoxylin and eosin, $\times 400$

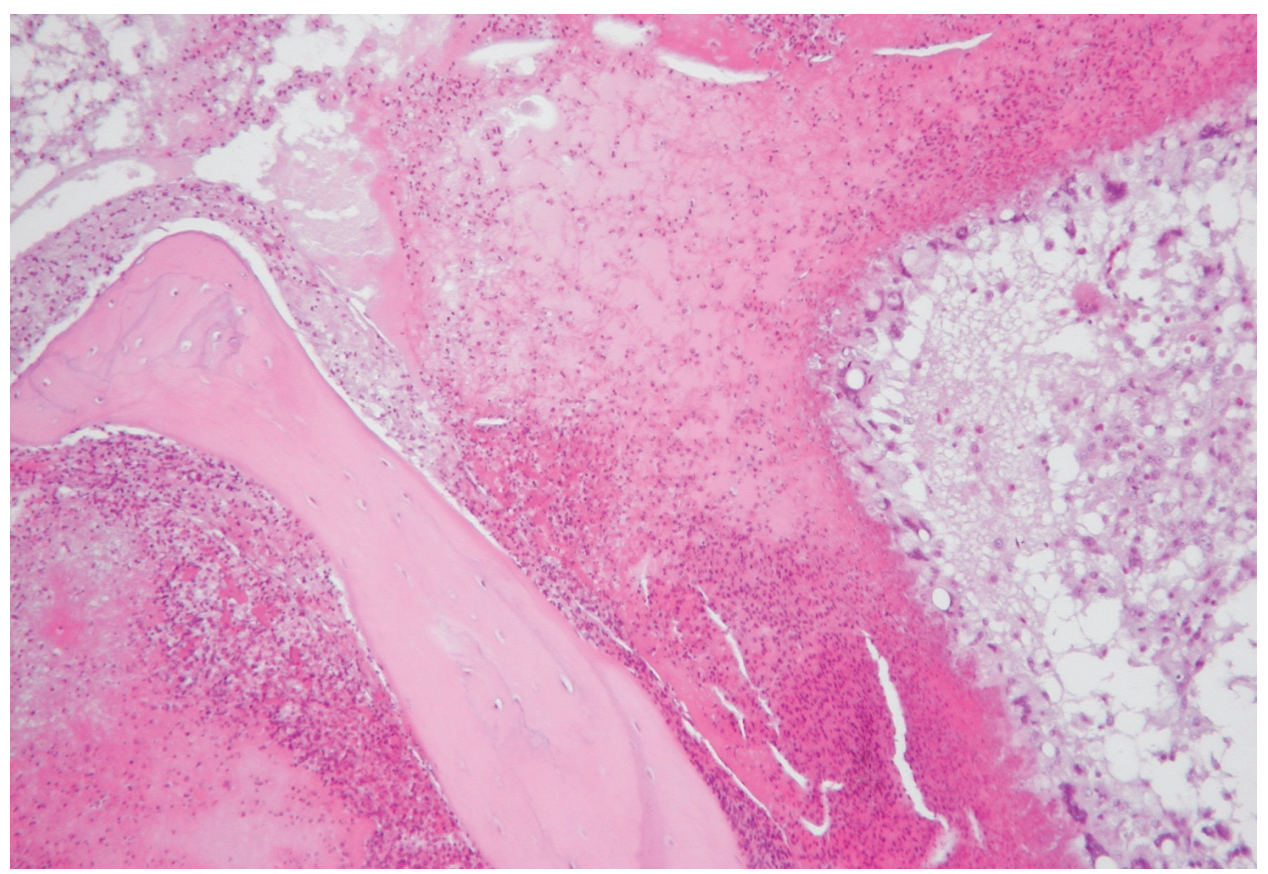

Fig. 9. Seropurulent osteomyelitis with heterophil granulocyte infiltration in the cranium of a turkey. Haematoxylin and eosin, $\times 200$ 


\section{Isolation of $\mathrm{R}$. anatipestifer}

The diagnosis of anatipestifer disease was always confirmed by the isolation of $R$. anatipestifer from the birds. The majority of strains from geese were isolated from the pericardium, brain, liver and occasionally from the joints, oviduct, lung, and in a few cases from the trachea, air sacs and peritoneum. The strains from ducks were most often isolated from the brain, pericardium or occasionally from the liver, oviduct, lung, trachea, and in one case from the conjunctiva. Almost all of the strains originating from turkeys were isolated from the cranium. In one case, $R$. anatipestifer was isolated from several organ samples in pure culture from the brain, air sac, trachea, pericardium, heart blood, liver, joint, and in mixed culture from the lungs.

\section{Discussion}

Over a period of five years, we examined 1418 cases of goslings, 411 cases of ducklings, and 1292 cases of turkeys. Our data show that anatipestifer disease was one of the most frequently diagnosed diseases in geese and ducks, while in turkeys it was found only occasionally.

In geese, $R$. anatipestifer infection typically occurs at the age of 5 days to 6 weeks (Riemer, 1904; Pierce and Vorhies, 1973; Ivanics et al., 1996; Hess et al., 2013). We have diagnosed the disease in older, up to 17-week-old birds as well. In other parts of the world, anatipestifer disease has been seldom reported in geese (Hess et al., 2013); however, in Hungary it is rather common, which may be explained by the large number and high density of geese flocks in certain areas of the country. In young birds, the gross lesions and histopathological findings obtained in our cases were generally similar to those previously reported elsewhere (Riemer, 1904; Pierce and Vorhies, 1973; Ivanics et al., 1996; Hess et al., 2013). Nevertheless, we have observed some differences. When lesions in the brain occurred, birds showed oedema and hyperaemia of the subcutaneous connective tissue over the cranium. These lesions were seen in 2- to 6-week-old geese, usually accompanied by serofibrinous meningitis and ventriculitis of the brain. We found the septicaemic form of the disease typically in 1- to 6-week-old geese. Riemerella anatipestifer was regularly isolated from the pericardium, brain, and liver.

At the age of 8 to 17 weeks, which is the usual force-feeding period of geese, generally catarrhal pneumonia was the only finding. Riemerella anatipestifer was isolated from the lung and occasionally from the air sacs. In waterfowl over 8 weeks of age, in conformity with data of the literature (Ruiz and Sandhu, 2013), $R$. anatipestifer infection occurred sporadically and produced only local lesions. In some rare cases, however, we observed the acute septicaemic form of anatipestifer disease even in 9- to 17-week old geese. The bacterium was 
isolated mostly from the liver, and occasionally from the pericardium, brain, lung and air sacs. Some of these birds had been force fed, which may have acted as a predisposing factor, or other immunosuppressive influences might have occurred. In about half of these cases, concurrent diseases were also observed.

In ducks, anatipestifer disease usually occurs at the age of 1 to 8 weeks (Pickrell, 1966; Jortner et al., 1969; Bitay et al., 1979). In our case, the disease was observed in 3- to 6.5-week-old ducks, which falls within this time interval. The gross and histopathological lesions were similar to those reported previously (Dougherty et al., 1955; Pickrell, 1966; Jortner et al., 1969; Bitay et al., 1979; Sarver et al., 2005), although we did not detect severe sinusitis, which had been described earlier in Hungary by Bitay et al. (1979). Oedema and hyperaemia of the connective tissue over the cranium were observed in the case of lesions in the brain, in the same way as found in geese. In one case, severe conjunctivitis was noticed in 3-week-old ducks, caused by $R$. anatipestifer infection in addition to mycoplasmosis suspected on the basis of the histopathological findings. The lack of septicaemia in ducks could possibly be explained by the lower pathogenicity of the bacterium or the different routes of exposure (Ruiz and Sandhu, 2013).

Riemerella anatipestifer is a facultative pathogen, and therefore anatipestifer disease occasionally occurs in combination with concurrent diseases both in waterfowl and turkeys (Ivanics et al., 1996; Banda et al., 2007; Rubbenstroth et al., 2009). In a certain part of the cases, $R$. anatipestifer infection complicated other primary diseases, such as circovirus infection, mycotoxicosis, mycoplasmosis, or Derzsy's disease. Circovirus causes immunosuppression in waterfowl; therefore, the presence of circovirus infection can facilitate several other infections. In our cases, the presence of circovirus infection, colibacillosis, paratyphoid, streptococcosis and pneumomycosis was detected within one case in addition to anatipestifer disease, which is consistent with the results of other authors (Ivanics et al., 1996; Banda et al., 2007; Rubbenstroth et al., 2009).

In turkeys, anatipestifer disease usually occurs at the age of 6 to 15 weeks (Zehr and Ostendorf, 1970; Helfer and Helmboldt, 1977; Smith et al., 1987). We have observed anatipestifer disease in turkeys at a slightly older age, at 12 to 19 weeks. Earlier studies reported that $R$. anatipestifer causes septicaemia, serofibrinous pericarditis, perihepatitis, airsacculitis, and occasionally pneumonia, sinusitis, arthritis and meningitis in turkeys. The isolation of this bacterium from the liver or spleen was usually successful (Zehr and Ostendorf, 1970; Helfer and Helmboldt, 1977; Smith et al., 1987). In contrast, we have diagnosed $R$. anatipestifer septicaemia in only one of the examined turkey cases. Generally, we observed local lesions such as purulent osteomyelitis in the cranium and seropurulent meningitis, arthritis and pericarditis mainly together with $E$. coli septicaemia or bacteraemia. American authors also observed E. coli infections that commonly followed $R$. anatipestifer infections in one to two weeks' time (Smith et al., 1987). In another case, serofibrinous pericarditis, peritonitis, purulent cranial os- 
teomyelitis, purulent meningitis, ventriculitis in the brain, and arthritis were seen in 15- to 18-week-old turkeys. Septicaemia was not observed in these birds, since $R$. anatipestifer was isolated from various organs but neither from the liver nor from the heart blood. Presumably, some immunosuppressive effects might have predisposed these birds to $R$. anatipestifer infection. Israeli authors also reported $R$. anatipestifer infection in turkeys without septicaemia (Frommer et al., 1990). They observed neurological signs besides slightly increased mortality, and $R$. anatipestifer was isolated from the brain of the diseased birds, but no gross pathological lesions could be found. American authors reported spondylitis in turkeys as a consequence of experimental infection with $R$. anatipestifer via the intravenous route performed one week after vaccination (Cooper and Charlton, 1992). Vertebral osteomyelitis (spondylitis) was found in the sixth thoracic vertebra; however, purulent osteomyelitis in the cranium in association with $R$. anatipestifer infection has not been reported in the literature so far. The explanation could be a previous bacteraemia or an initiated antibiotic therapy. The origin of $R$. anatipestifer infection could not be thoroughly traced; however, transmission from duck populations kept in the region cannot be excluded. Previous studies reported nearby duck farms or duck manure as a source of $R$. anatipestifer infection in turkeys (Zehr and Ostendorf, 1970; Helfer and Helmboldt, 1977; Smith et al., 1987; Frommer et al., 1990).

The high incidence of the disease in waterfowl underlines the importance of appropriate treatment and prevention. Antibiotics are important tools for controlling the disease. On the basis of the antibiotic susceptibility testing of $185 R$. anatipestifer strains isolated in Hungary, the most effective antibiotics were florfenicol, ampicillin, penicillin and sulphamethoxazole-trimethoprim with $97.9 \%$, $95.1 \%, 93 \%$ and $92.4 \%$ sensitivity, respectively, although the resistance patterns showed some variation depending on the geographical origin and the year of isolation (Gyuris et al., 2017). Riemerella anatipestifer is a facultative pathogen, and therefore predisposing factors such as unfavourable environmental conditions should be avoided and concomitant diseases should be treated. Proper management procedures such as good sanitation practices, high level of biosecurity (separation of flocks on multiple-age farms, keeping away wild waterfowl and rodents) and all-in/all-out production should be implemented (Ruiz and Sandhu, 2013). Inactivated autogenous vaccines can be used for prevention (Ivanics et al, 1996). This approach seems to be the most effective as no cross-protection has been observed between the 21 serotypes of $R$. anatipestifer (Ruiz and Sandhu, 2013). Regular monitoring of the actual serotypes in a flock is recommended because more than one serotype can be present or the serotypes can change over time in the same farm (Bisgaard et al., 2008; Ryll et al., 2001). 


\section{Acknowledgements}

The authors thank Éva Ivanics, Ákos Thuma and Andrea Tünde Bistyák for making available the reports and documents of their cases, Róbert Glávits for the excellent advice, and Judit Markovics, Angéla Kempa-Tóth, Ildikó Képíróné Szabó, Szilvia Lakosi and Ágnes Mészáros for their skilful technical assistance. This work was supported by the National Research, Development and Innovation Office - NKFIH K108632.

\section{References}

Banda, A., Galloway-Haskins, R. I., Sandhu, T. S. and Schat, K. A. (2007): Genetic analysis of a duck circovirus detected in commercial Peking ducks in New York. Avian Dis. 51, 90-95.

Bisgaard, M., Bojesen, A. M. and Christensen, J. P. (2008): Riemerella infections. In: Pattison, M., McMullin, P. F., Bradbury, J. M. and Alexander, D. J. (eds) Poultry Diseases. Elsevier Press, Edinburgh, UK. pp. 172-175.

Bitay, Z., Kovács, Gy., Takács, Gy. and Török, L. (1979): Occurrence of the anatipestifer syndrome of ducks in Hungary (Pasteurella anatipestifer bacteria) [in Hungarian, with English abstract]. Magy. Allatorvosok 34, 747-750.

Bruner, D. W., Angstrom, C. I. and Price, J. I. (1970): Pasteurella anatipestifer infection in pheasants. A case report. Cornell Vet. 60, 491-494.

Cooper, G. L. and Charlton, B. R. (1992): Spondylitis in turkeys associated with experimental Pasteurella anatipestifer infection. Avian Dis. 36, 290-295.

Dougherty, E., Saunders, L. Z. and Parsons, E. H. (1955): The pathology of infectious serositis of ducks. Am. J. Pathol. 31, 475-487.

Frommer, A., Bock, R., Inbar, A. and Zemer, S. (1990): Muscovy ducks as a source of Pasteurella anatipestifer infection in turkey flocks. Avian Pathol. 19, 161-163.

Fulton, R. M. and Rimler, R. B. (2010): Epidemiologic investigation of Riemerella anatipestifer in a commercial duck company by serotyping and DNA fingerprinting. Avian Dis. 54, 969-972.

Gyuris, É., Wehmann, E., Czeibert, K. and Magyar, T. (2017): Antimicrobial susceptibility of Riemerella anatipestifer strains isolated from geese and ducks in Hungary. Acta Vet. Hung. 65, $153-165$.

Helfer, D. H. and Helmboldt, C. F. (1977): Pasteurella anatipestifer infection in turkeys. Avian Dis. 21, 712-715.

Hendrickson, J. M. and Hilbert, K. F. (1932): A new and serious septicemic disease of young ducks with a description of the causative organism, Pfeifferella anatipestifer. N. S. Cornell Vet. 22, 239-252.

Hess, C., Enichlmayr, H., Jandreski-Cvetkovic, D., Liebhart, D., Bilic, I. and Hess, M. (2013): Riemerella anatipestifer outbreaks in commercial goose flocks and identification of isolates by MALDI-TOF mass spectrometry. Avian Pathol. 42, 151-156.

Hinz, K. H., Ryll, M., Köhler, B. and Glünder, G. (1998): Phenotypic characteristics of Riemerella anatipestifer and similar micro-organisms from various hosts. Avian Pathol. 27, 33-43.

Ivanics, É., Glávits, R. and Édes, I. (1996): A study on the anatipestifer disease of growing geese [in Hungarian, with English abstract]. Magy. Allatorvosok 51, 9-14.

Jortner, B. S., Porro, R. and Leibovitz, L. (1969): Central-nervous-system lesions of spontaneous Pasteurella anatipestifer infection in ducklings. Avian Dis. 13, 27-35.

Kardos, G., Nagy, J., Antal, M., Bistyák, A., Tenk, M. and Kiss, I. (2007): Development of a novel PCR assay specific for Riemerella anatipestifer. Lett. Appl. Microbiol. 44, 145-148.

Karstad, L., Lusis, P. and Long, J. R. (1970): Pasteurella anatipestifer as a cause of mortality in captive wild waterfowl. J. Wildl. Dis. 6, 408-413. 
Pascucci, S., Giovannetti, L. and Massi, P. (1989): Pasteurella anatipestifer infection in guinea fowl and Japanese quail (Coturnix coturnix japonica). Proceedings of the 9th International Congress of the World Veterinary Poultry Association, Brighton, England. p. 47.

Pickrell, J. A. (1966): Pathologic changes associated with experimental Pasteurella anatipestifer infection in ducklings. Avian Dis. 10, 281-288.

Pierce, R. L. and Vorhies, M. W. (1973): Pasteurella anatipestifer infection in geese. Avian Dis. 17, 868-870.

Riemer von, S. (1904): Kurze Mitteilung über eine bei Gänsen beobachtet exsudative Septikämie und deren Erreger. Zentralbl. Bakteriol. I Abt. I Orig. 37, 641-648.

Rosenfeld, L. E. (1973): Pasteurella anatipestifer infection in fowls in Australia. Aust. Vet. J. 49, $55-56$.

Rubbenstroth, D., Ryll, M., Behr, K. P. and Rautenschlein, S. (2009): Pathogenesis of Riemerella anatipestifer in turkeys after experimental mono-infection via respiratory routes or dual infection together with the avian metapneumovirus. Avian Pathol. 38, 497-507.

Ruiz, J. A. and Sandhu, T. S. (2013): Riemerella anatipestifer infection. In: Saif, Y. M. (ed.) Diseases of Poultry. Iowa State University Press, Iowa, USA. pp. 823-828.

Ryll, M., Christensen, H., Bisgaard, M., Christensen, J.-P., Hinz, K.-H. and Köhler, B. (2001): Studies on the prevalence of Riemerella anatipestifer in the upper respiratory tract of clinically healthy ducklings and characterization of untypable strains. J. Vet. Med. 48, 537-546.

Sarver, C. F., Morishita, T. Y. and Nersessian, B. (2005): The effect of route of inoculation and challenge dosage on Riemerella anatipestifer infection in Pekin ducks (Anas platyrhynchos). Avian Dis. 49, 104-107.

Segers, P., Mannheim, W., Vancanneyt, M., de Brandt, K., Hinz, K. H., Kersters, K. and Vandamme, P. (1993): Riemerella anatipestifer gen. nov., comb. nov., the causative agent of septicemia anserum exsudativa, and its phylogenetic affiliation within the Flavobacterium-Cytophaga rRNA homology group. Int. J. Syst. Bacteriol. 43, 768-776.

Smith, J. M., Frame, D. D., Cooper, G., Bickford, A. A., Ghazikhanian, G. Y. and Kelly, B. J. (1987): Pasteurella anatipestifer infection in commercial meat-type turkeys in California. Avian Dis. 31, 913-917.

Zehr, W. J. and Ostendorf, J. (1970): Pasteurella anatipestifer in turkeys. Avian Dis. 14, 557-560. 